\title{
Die Europäischen Gewerkschafts- verbände: Zur Entwicklung ihrer Machtressourcen und Funktionsprofile
}

Die vor zehn Jahren einsetzende Weltfinanzmarkt- und Euro-Krise stellte die Handlungsfähigkeit der Europäischen Gewerkschaftsverbände (EGV) vor gewaltige Herausforderungen. Die national unterschiedlichen Krisenerfahrungen und die wachsende Heterogenität nationaler Gewerkschaftsinteressen erschwerten die Verfolgung gemeinsamer europäischer Handlungsstrategien. Gleichzeitig veränderte sich mit dem neuen System der Europäischen Economic Governance der institutionelle Handlungskontext der EGV auf europäischer Ebene. Vor diesem Hintergrund untersucht dieser Beitrag, wie sich die Machtressourcen, Funktionsprofile und Politiken der Europäischen Gewerkschaftsverbände im Verlauf der Krise verändert haben.

HANS-WOLFGANG PLATZER, TORSTEN MÜLLER

\section{Einleitung}

In den Gewerkschaften wie in der Wissenschaft wird kontrovers darüber diskutiert, wie eine dem Binnenmarkt, der Währungsunion und dem EU-Mehrebenensystem des Regierens „angemessene“ Europäisierung gewerkschaftlicher Organisationsformen und Handlungsstrategien beschaffen sein sollte.

Im gegenwärtigen krisengeschüttelten Europa stellen sich, zehn Jahre nach dem Ausbruch der Weltfinanzmarktkrise, die in eine „Eurokrise“ mündete, neue und schwierige Fragen. So ist zu klären, ob und inwieweit die krisenhaften Entwicklungen zu einem Auseinanderdriften der je nationalen gewerkschaftlichen Machtressourcen, Handlungsbedingungen und politischen Prioritäten geführt oder umgekehrt grenzübergreifende und ähnliche Problemlagen hervorgebracht haben, die gemeinsame europäische Handlungsansätze begünstigen können und realiter auch befördert haben.

Um solche Spannungsfelder empirisch detailliert auszuloten, wäre es erforderlich, eine vergleichende Analyse der nationalen Gewerkschaften und Arbeitsbeziehungen in der EU-28 (vgl. Müller/Platzer 2016) mit einer Analyse der grenzübergreifenden und überstaatlichen gewerkschaftlichen Organisation und Politik zu verbinden.

Die nachfolgende Analyse beschränkt sich auf die europäisch-transnationale Ebene (vgl. Müller/Platzer 2018).
Sie wirft einen Blick auf die längerfristigen Entwicklungslinien der Europäischen Gewerkschaftsverbände (EGV) und fragt, ob und inwieweit sich deren Machtressourcen, Aufgaben und Politiken im vergangenen Krisenjahrzent verändert haben, wie sie sich gegenwärtig darstellen und welche künftigen Anforderungen die gewerkschaftliche Politik auf europäischer Ebene zu bewältigen hat.

Als theoretisches Gerüst dient die Verknüpfung zweier Ansätze, die die Autoren an anderer Stelle entwickelt haben: ein Modell gewerkschaftlicher Machtressourcen in einem europäischen Mehrebenensystem der Arbeitsbeziehungen (Müller/Platzer 2016) und eine Typologie transnationaler Gewerkschaftszusammenschlüsse, die es ermöglicht, unterschiedliche Entwicklungsstufen, Funktionsprofile und Akteursqualitäten der EGV zu erfassen (Platzer/Müller 2009).

\section{Theoretischer Rahmen}

2.1 Gewerkschaftliche Machtressourcen im europäischen Mehrebenensystem

Die jüngere Gewerkschaftsforschung analysiert die Rolle und Macht von Gewerkschaften mittels eines Machtressourcenansatzes (vgl. Haipeter/Dörre 2011; Schmalz/ Dörre 2013). In der Regel werden vier Machtquellen 


\section{Gewerkschaftliche Machtressourcen im europäischen Mehrebenensystem der Arbeitsbeziehungen}

\begin{tabular}{|c|c|c|}
\hline & \multicolumn{2}{|c|}{ Quellen und Komponenten gewerkschaftlicher Macht } \\
\hline & Nationale Ebene & Transnational-europäische Ebene \\
\hline $\begin{array}{l}\text { Ökonomisch- } \\
\text { strukturelle } \\
\text { Macht }\end{array}$ & Arbeitsmarkt- und Produktionsmacht & $\begin{array}{l}\text { - Arbeitsmarkt- und Produktionsmacht in transnationalen Wertschöpfungsprozessen; } \\
\text { - grenzübergreifende (positive oder negative) Effekte der national unterschiedlichen Marktmacht } \\
\text { innerhalb der mitgliedstaatlichen Arbeitsmarkt- und Produktionsmodelle }\end{array}$ \\
\hline $\begin{array}{l}\text { Organisations- } \\
\text { macht }\end{array}$ & $\begin{array}{l}\text { Mitgliederstärke, Stabilität und Vitalität } \\
\text { gewerkschaftlicher Organisation }\end{array}$ & $\begin{array}{l}\text { - Fähigkeit zur transnationalen Bündelung nationaler und lokaler Machtmittel; } \\
\text { - grenzüberschreitende Vernetzung entlang der Wertschöpfungsketten und länderübergreifende } \\
\text { Unterstützungsleistungen stärkerer an schwächere Gewerkschaften; } \\
\text { - Ressourcen- und Kompetenzausstattung der europäischen Verbandsebene }\end{array}$ \\
\hline $\begin{array}{l}\text { Institutionelle } \\
\text { Macht }\end{array}$ & $\begin{array}{l}\text { Sicherung von Einfluss } \\
\text { in institutionellen Arrangements }\end{array}$ & $\begin{array}{l}\text { - Sicherung von Einfluss in der EU: } \\
\text { auf vertraglicher Grundlage bzw. in den durch EU-Recht geschaffenen europäischen Arenen }\end{array}$ \\
\hline $\begin{array}{l}\text { Kommunikative } \\
\text { Macht }\end{array}$ & $\begin{array}{l}\text { Diskursfähigkeit und Meinungsführerschaft } \\
\text { sowie die Fähigkeit zu Bündnissen } \\
\text { mit anderen zivilgesellschaftlichen Akteuren }\end{array}$ & $\begin{array}{l}\text { - Kohärenz ideologisch-programmatischer Positionen; } \\
\text { - Vermittlungsfähigkeit europapolitischer Strategievorstellungen gegenüber den EU-Organen; } \\
\text { - Fähigkeit zu Bündnissen mit transnationalen Nichtregierungsorganisationen }\end{array}$ \\
\hline
\end{tabular}

identifiziert, die sich teilweise gegenseitig bedingen und ergänzen, bis zu einem gewissen Grade auch gegenseitig ersetzen können: die ökonomisch-strukturelle Macht, die Organisationsmacht, die institutionelle Macht und die kommunikative Macht.

Dieses Machtressourcenmodell und die entsprechende Machtanalytik sind auf einen nationalstaatlichen Rahmen bezogen. Unterbelichtet bleiben hingegen die zunehmenden politischen Verflechtungen und sozial-ökonomischen Interdependenzen im europäischen Integrationsraum und die wachsende Bedeutung der europäischen Entscheidungsebene.

Demzufolge gilt es, den Machtressourcenansatz um eine europäisch-transnationale Dimension zu erweitern, denn

- alle vier oben genannten national lokalisierten Machtquellen unterliegen trans- und supranationalen ökonomischen wie politischen Einflüssen. Diese Einflüsse können zu einer Schwächung, unter bestimmten Umständen auch Stärkung, der je nationalen Machtressourcen führen;

- politische Entscheidungen auf europäischer Ebene beeinflussen zwischenstaatlich wie zwischengewerkschaftlich die Wettbewerbsparameter und damit (mittelbar und unmittelbar) die je nationalen und transnationalen Machtressourcen;

- eine genuin europäisch-transnationale Ebene ökonomisch-struktureller, organisatorischer, institutioneller und kommunikativer Machtressourcen gewinnt an Bedeutung.

Aus diesen Vorüberlegungen lässt sich das nachstehende europäische Mehrebenen-Modell gewerkschaftlicher Machtressourcen entwickeln und für die empirische Ana- lyse fruchtbar machen. Im vorgegebenen Rahmen sind hierbei zwei Einschränkungen unvermeidlich: Theoretisch kann lediglich das Grundgerüst skizziert werden und in der nachfolgenden empirischen Analyse können wiederum nur einzelne Machtdimensionen mit Fokus auf die europäische Ebene und die Funktionsprofile der Europäischen Gewerkschaftsverbände eingehender beleuchtet werden.

Für dieses europäische Mehrebenen-Modell gewerkschaftlicher Machtressourcen kann die nationale Ebene im Anschluss an vorliegende Arbeiten (Huke/Tietje 2015, S. 374ff.) wie folgt beschrieben werden:

(1) Die ökonomisch-strukturelle Macht von Gewerkschaften als kollektive Interessenvertretungen abhängig Beschäftigter wird durch die Situation Lohnabhängiger auf dem Arbeitsmarkt und durch deren spezifische Stellung im Produktionsprozess bestimmt. Diese „Marktmacht“ beruht auf der Fähigkeit, Produktions-und Kapitalverwertungsprozesse zu beeinflussen bzw. „zu stören“. Dabei spielen wiederum die folgenden Parameter eine Rolle: Qualifikationsniveau, Substituierbarkeit der Arbeit, Segmentierung des Arbeitsmarktes in Stammbelegschaften und prekär Beschäftigte sowie die Arbeitslosenquote.

(2) Die ökonomisch-strukturelle Machtquelle kommt freilich erst dann zum Tragen, wenn sie mit Organisationsmacht verbunden ist, die sich aus den kollektiven Zusammenschlüssen der abhängig Beschäftigten zu Gewerkschaften ergibt. Organisationsmacht ist zunächst numerisch und materiell definiert: durch Mitgliederzahlen und Organisationsgrade und die damit verbundenen Finanz- und Personalausstattungen einschließlich der „Streikkassen“ der Verbände. Darüber hinaus ist Organi- 
sationsmacht aber auch eine qualitative Größe, bei der es um spezifische Fähigkeiten zur Mitgliedermobilisierung und zum kollektiven, strategischen Handeln sowie um die Vitalität und Attraktivität der Organisation geht.

(3) Die institutionelle Macht ist eine gleichsam „sekundäre“, aber gewichtige Machtquelle der Lohnabhängigen und Gewerkschaften. Sie stellt eine historisch "geronnene“ Machtform dar, die aus Arbeitskämpfen und sozialen Kompromissen zwischen Unternehmen, Staat und Gewerkschaften hervorgegangen ist. Sie drückt sich in institutionellen Formen wie dem Arbeitsrecht, dem Wohlfahrtsstaat, der Mitbestimmung und dem Tarifsystem ebenso aus wie in bestimmten kulturellen Traditionen und Praktiken des Systems der Arbeitsbeziehungen. Institutionelle Macht sichert die gewerkschaftliche Partizipation und stabilisiert bzw. erleichtert die gewerkschaftliche Interessenartikulation ohne eine permanente „Mobilisierungspflicht“.

(4) Die kommunikative Macht beschreibt zum einen gewerkschaftliche Fähigkeiten, ad hoc erfolgreich in öffentliche Debatten eingreifen zu können und (im Idealfall) die Meinungsführerschaft und Diskurshoheit zu erringen, zum andern eine längerfristige Präsenz und Wirksamkeit gewerkschaftlicher Ideen im gesellschaftlich-öffentlichen Raum. Teil der kommunikativen Macht ist schließlich die Fähigkeit, bei der Beeinflussung der öffentlichen und veröffentlichten Meinung mit anderen zivilgesellschaftlichen Akteuren zu kooperieren. ${ }^{1}$

Auf der europäisch-transnationalen Ebene können die gewerkschaftlichen Machtquellen ebenfalls in diesen vier Dimensionen abgebildet (Übersicht 1) und - in gebotener Kürze - wie folgt charakterisiert werden:

(1) Die transnationale ökonomisch-strukturelle Macht kann in transnational stark verflochtenen Produktionsprozessen und hoch integrierten Märkten (unmittelbar) wirksam werden, in denen eine grenzübergreifende „Macht zu stören“ gegeben ist. Mittelbar (teilweise auch unmittelbar) wird diese Machtdimension durch die Unterschiede in den mitgliedstaatlichen Arbeitsmarkt- und Produktionsmodellen beeinflusst, da eine darin verankerte gewerkschaftliche Primär- oder Marktmacht Auswirkungen auf den grenzübergreifenden Wettbewerb im Binnenmarkt („Standortkonkurrenz“) und in der Währungsunion (Probleme eines „Lohndumping“) hat.

(2) Die transnationale Organisationsmacht baut auf der jeweiligen nationalen und lokalen Macht auf. Bedeutsam sind deshalb die Bereitschaft zu solidarischen transnationalen Unterstützungsleistungen und die Fähigkeit der Gewerkschaften, über nationale Grenzen hinweg - zumal entlang von Wertschöpfungsketten - zusammenzuarbeiten und in variablen Netzwerken transnationale Gegenmacht zu entfalten. Schließlich manifestiert sich die trans- nationale Organisationsmacht in der Ressourcen- und Kompetenzausstattung der europäischen Verbandsebene.

(3) Die transnationale institutionelle Macht besteht in der Ermöglichung und Sicherung von gewerkschaftlichem Einfluss in europäischen Arenen, die EU-vertraglich verankert sind (wie bspw. der [multi-]sektorale Soziale Dialog) bzw. die durch EU-Recht geschaffen sind (wie z. B. der Europäische Betriebsrat bzw. der SE-Betriebsrat). Ein weiteres Beispiel in dieser Machtdimension wäre - im Falle einer europarechtlichen Verabschiedung und Umsetzung - ein (optionaler) Rechtsrahmen für eine transnationale unternehmensbezogene Vereinbarungspolitik und zwar (nur) dann, wenn ein solches Regelwerk die gewerkschaftliche Prärogative in den transnationalen Tarifverhandlungen sichern würde. Die transnationale institutionelle Machtdimension schließt freilich auch jene negativen Effekte ein, die aus politischen Entscheidungen auf europäischer Ebene resultieren (können), welche zugleich die nationale gewerkschaftliche Machtbasis schwächen (können). Seien dies technokratische Entscheidungen und ein supranationaler lohnpolitischer Interventionismus (Schulten/Müller 2015), wie er in den „Programmländern“ durch die Troika exekutiert wurde, oder sei dies die krisenbedingte Verstärkung und längerfristige Verfestigung einer auf europäischer Ebene gesteuerten Konsolidierungspolitik, die nationale institutionelle Arrangements zulasten der Gewerkschaften verändert.

(4) Die transnationale kommunikative Macht ist eine Machtquelle, die nicht in erster Linie auf eine europäische öffentliche oder veröffentlichte Meinung zielt, da diese nach wie vor schwach ausgeprägt ist und Europapolitik noch immer überwiegend in nationalen Diskursräumen verhandelt wird. Vielmehr geht es hier um die Kohärenz ideologisch-programmatischer Positionen und um die Fähigkeit, europapolitische Interessen und Strategievorstellungen gleichgerichtet in den je nationalen Handlungskontexten wie auf der europäischen Ebene zu vermitteln. Die Kohärenz und Substanz gemeinsamer europäischer Politikentwürfe und Regulierungsvorstellungen ist auch eine wichtige Vorbedingung für erfolgreiche Vernetzungsaktivitäten und für die Schaffung von Bündnissen ( $a d$ vocacy coalitions) innerhalb einer vielfältigen „Brüsseler Lobby-Szene“.

\subsection{Funktionsprofile}

transnationaler Gewerkschaftsverbände

Nationale Gewerkschaften können in unterschiedlicher thematischer Reichweite und politischer Verbindlichkeit

1 Huke/Tietje (2015, S. 374f.) fassen diese beiden Teile als Kooperationsmacht und Diskursmacht unter der Kategorie der gesellschaftlichen Macht zusammen. 
ihre Interessen transnational koordinieren und vermitteln und dafür ihre europäische Organisationsebene mit unterschiedlichen Ressourcen und Kompetenzen ausstatten. Ausgehend von diesen Vorüberlegungen (vgl. Platzer/ Müller 2009, S. 31fff.) lässt sich das nachfolgende Stufenmodell (in absteigender Reihenfolge) entwickeln (Übersicht 2).

Dieses „idealtypisierende“ taxonomische Raster ermöglicht ebenso eine entwicklungsgeschichtliche Beschreibung der Verbände wie einen Vergleich ihrer gegenwärtigen Funktionsprofile und Akteurseigenschaften. Dieses Stufenmodell bedarf (was hier nur erwähnt, aber nicht ausgeführt werden kann) für die empirische Feinanalyse einer Ausdifferenzierung nach Aufgabenbereichen, da z. B. ein EGV im Handlungsfeld der Konzernpolitik Merkmale eines Steuerungsverbundes aufweisen kann, während der gleiche EGV im Handlungsfeld der tarifpolitischen Koordinierung als Forum fungiert.

\section{Empirische Befunde}

\subsection{Die Funktionsprofile der EGV im historischen Längsschnitt}

Im historischen Längsschnitt und im Vergleich zu den Profilen der transnationalen Verbände in den ersten Jahrzehnten nach Gründung der heutigen EU, die meist den Charakter von runden Tischen und Foren hatten, hat sich die transnationale Zusammenarbeit innerhalb der EGV im Laufe der 1980er und 9oer Jahre sukzessive verdichtet, thematisch erweitert und politisch intensiviert.

Neben der Fortschreibung von Verbandsroutinen im Bereich der mitgliederbezogenen Politik (Öffentlichkeitsarbeit, Informationsdienstleistungen, Schulungsaktivitäten etc.) haben sich mit der Ausbildung neuer transnationaler Handlungsfelder und -instrumente zugleich die Funktionsprofile der Verbände verändert. Insbesondere in den Bereichen der tarifpolitischen Koordinierung (die mit Einführung des Euro eine neue strategische Bedeutung erhielt), der Konzernpolitik (die mit der 1994 verabschiedeten Europäischen Betriebsräte-Richtlinie ein neues transnationales Handlungsfeld eröffnete) und der arbeitspolitischen Selbstregulierung im Rahmen der Sektoralen Sozialdialoge nutzen die Mitgliedsverbände den europäischen Organisationsrahmen als „Ort" einer zunehmend verbindlicheren und thematisch konkreteren Interessenabstimmung und Handlungskoordination. Im Zuge dieses Prozesses haben sich die Verbände mehrheitlich von Foren zu Koordinierungsplattformen entwickelt. Bei einzelnen Verbänden hat sich in bestimmten Themenfeldern das Funktionsprofil eines transnationalen Steuerungsverbundes herausgebildet.

\section{ÜBERSICHT 2}

\section{Funktionsprofile transnationaler Gewerkschaften}

\begin{tabular}{|c|c|}
\hline Funktionsprofil & Modus und Reichweite transnationaler Problembearbeitung \\
\hline Supranationaler Verband & $\begin{array}{l}\text { - Hierarchische Steuerung } \\
\text { - umfassende Kompetenzen und Mandatierung; } \\
\text { kontinuierliche eigenständige Wahrnehmung } \\
\text { gewerkschaftlicher Kernfunktionen z. B. in den Bereichen } \\
\text { der Tarif- und Unternehmenspolitik }\end{array}$ \\
\hline Steuerungsverbund & $\begin{array}{l}\text { - Festlegung verbindlicher Entscheidungen (Joint Decisions) } \\
\text { - Standardisierung operativer Ziele (z. B. tarif- und/oder } \\
\text { unternehmenspolitische Koordinierungsregeln) } \\
\text { - fallweise sowie thematisch und zeitlich begrenztes Mandat } \\
\text { für transnationale Verhandlungen } \\
\text { - Festlegung von Mechanismen zur Implementierung } \\
\text { und Überwachung von Entscheidungen } \\
\text { - Herstellung von Verbindlichkeit und verbandsinterner Transparenz }\end{array}$ \\
\hline Koordinierungsplattform & $\begin{array}{l}\text { - Aushandlung unverbindlicher Entscheidungen } \\
\text { - geregelte und strukturierte Abstimmung } \\
\text { über gemeinsame Positionen } \\
\text { - Standardisierung operativer Ziele (z. B. Mustervereinbarungen) } \\
\text { - Positionsabstimmung mit „weicher“ Orientierungsfunktion } \\
\text { für Mitgliedsverbände }\end{array}$ \\
\hline Forum & $\begin{array}{l}\text { - geregelter Informationsaustausch } \\
\text { - kontinuierliche Positionsabstimmung und -vermittlung } \\
\text { - verständigungsorientierte Annäherung }\end{array}$ \\
\hline Informationsdienstleister & $\begin{array}{l}\text { - Informationsaustausch } \\
\text { - partielle bzw. thematisch begrenzte Kooperation } \\
\text { und Positionsabstimmung }\end{array}$ \\
\hline
\end{tabular}

Quelle: Eigene Darstellung

Diese allgemeine Entwicklungstendenz wird allerdings mehrfach gebrochen: Zunächst zeigen sich bei allen Verbänden Widersprüche zwischen „Deklaration“ und „Praxis“. Dies bedeutet, dass die Verständigungsprozesse, etwa im Bereich der Tarif- und Konzernpolitik, zwar zur Festlegung verbindlicher Ziele und Verfahrensregeln auf europäischer Ebene führen, die Einhaltung der europäisch vereinbarten Politiken, die ja von der Selbstverpflichtung der Mitgliedsverbände abhängt, letztlich jedoch an deren dezentraler Durchsetzungsmacht in den jeweils nationalen Kontexten scheitert.

Des Weiteren variieren die Intensitätsgrade der Handlungskoordinierung innerhalb eines Verbandes sowohl auf der Zeitachse als auch nach Themenbereichen und Handlungsfeldern. Schließlich sind auch die Unterschiede zwischen den EGV - jenseits der oben beschriebenen allgemeinen Transnationalisierungstendenzen - nach wie vor beträchtlich.

Die graduelle Erweiterung und Stärkung der Funktionsprofile der EGV kommt nicht allein in der Stärkung der transnationalen Sekretariatsressourcen zum Ausdruck. Das im Zuge der vertieften EU-Marktintegration komplexere und anspruchsvollere Management of Interdependence gewerkschaftlicher Interessen und die im Zuge der EU-Osterweiterung gleichzeitig gewachsenen und schwierigeren Anforderungen eines Management of 
Diversity haben bei keinem Europäischen Gewerkschaftsverband dazu geführt, auf transnationaler Sekretariatsebene die professionellen Ressourcen zu bündeln und zu erweitern oder die Entscheidungsprozesse auf überstaatlicher Ebene zu zentralisieren. Vielmehr dominiert bei allen EGV ein Politikansatz, wonach von nationalen Gewerkschaftsexperten getragene Ausschüsse oder Task Forces die zentralen Organisations- und Arbeitseinheiten der transnationalen Verbände bilden.

\subsection{Entwicklung der transnationalen Machtressourcen im jüngsten Krisenjahrzehnt}

Eine solidarische transnationale Politik der Gewerkschaften und eine effektive Interessendurchsetzung auf europäischer Ebene ist umso eher möglich, je kohärenter und konvergenter die je nationalen gewerkschaftlichen Interessenlagen und die national mobilisierbaren Machtressourcen sind und je mehr die EU-Politik institutionelle Machtressourcen - zum Beispiel durch eine gewerkschaftsfreundliche regulative Politik - bereitstellt. Betrachtet man unter diesen Prämissen den Krisenverlauf, so ergibt sich mit Blick auf die transnationalen gewerkschaftlichen Machtressourcen das folgende Bild:

3.2.1 Transnationale ökonomische Machtressourcen Die krisenbedingten Veränderungen der transnationalen ökonomisch-strukturellen Macht der Gewerkschaften sind aus empirisch-methodischen Gründen analytisch schwer zu fassen, weil die mit den nationalen Veränderungen der gewerkschaftlichen Marktmacht verbundenen transnationalen Effekte - Standortwettbewerb, sozial-und arbeitspolitisches downsizing, Lohndumping etc. - nur annäherungsweise bestimmbar sind. Empirisch evident ist, dass in transnational stark verflochtenen Produktions- oder Dienstleistungsbereichen, in denen grundsätzlich eine grenzübergreifende „Macht zu stören“ gegeben wäre, diese nicht mobilisiert werden konnte. Ein wesentlicher Grund hierfür ist, dass trotz gemeinsamer Probleme und einer länderübergreifenden Krisendynamik die mitgliedstaatlichen Krisenverläufe und die Folgen im Bereich der Arbeitsmärkte und der Arbeitsbeziehungen doch sehr unterschiedlich waren und sich zwischen zwei Polen bewegten:

In Ländern, die dem nordischen Korporatismus bzw. dem kontinentaleuropäischen Sozialpartnerschaftsmodell angehören (Österreich, Deutschland, die nordischen Länder), gelang es, die Krise mittels eines „Krisenkorporatismus" abzufangen und zu moderieren. Dabei waren die Gewerkschaften aktiv in das Krisenmanagement einbezogen. In den Tauschprozessen waren sie zwar zu teilweise schmerzhaften Konzessionen gezwungen, konnten aber auch eigene Interessen erfolgreich durchsetzen - in Deutschland etwa Arbeitsplatzgarantien, die Verlängerung des Kurzarbeitergeldes oder die Mitgestaltung der betriebsinternen Arbeitszeitflexibilisierung. In den Län- dern des südlichen Europa sowie in einigen mittel- und osteuropäischen Ländern war ein solcher Problemlösungspfad hingegen blockiert, weil entsprechende Arbeitsbeziehungstraditionen fehlten oder die Wucht der Krise bestehende Aushandlungsmechanismen geschwächt und vielfach gesprengt hat. In den „Krisen- bzw. Programmländern“ haben nicht zuletzt exogene Faktoren, insbesondere die Politik der Troika, Entwicklungen befördert (Druck auf Flächentarife, Absenkung der Mindestlöhne etc.), die die je nationale gewerkschaftliche Machtbasis vor allem im Bereich der Organisationsmacht und institutionellen Macht - geschwächt haben.

\subsubsection{Transnationale}

politisch-institutionelle Machtressourcen

Beleuchtet man mit Hilfe des theoretischen Machtressourcenmodells die transnationale Dimension, so ist vor allem die Kategorie der institutionellen Machtressourcen bedeutsam, weil hier auf europäischer Ebene durch die Krisenprozesse seit 2008 weitreichende Weichen gestellt und neue Institutionen und Steuerungsformen etabliert wurden; darunter der Europäische Stabilitätsmechanismus, der Fiskalpakt, die EU-Regulierungen des sogenannten Six-Pack und Two-Pack, das Europäische Semester sowie die im Entstehen begriffene Bankenunion. Das neue Instrumentarium der Economic Governance verändert die Rahmenbedingungen gewerkschaftlichen Handelns auf je nationaler und transnationaler Ebene, weil Löhne und Lohnfindungssysteme zu einer zentralen Anpassungsvariable bei makro-ökonomischen Ungleichgewichten werden. Das neue System hat vor allem in den Anfangsjahren der Krise und besonders ausgeprägt in den „Programmländern“ die Erosion bestehender Flächentarifsysteme sowie Lohnsenkungen befördert.

Hingegen sind hinsichtlich der gewerkschaftlich relevanten „sozialen Dimension“ der EU, also in den Bereichen der sozialpolitischen Regulation (EU-Gesetzgebung), der Distribution (Einsatz spezifischer Finanzmittel), des Sozialen Dialogs und der Offenen Methode der Koordinierung im Zuge der Krisenbearbeitung nur bescheidene Neuerungen zu verzeichnen: In Reaktion auf die Jugendarbeitslosigkeit kam es nach längerem Vorlauf auf EU-Ebene zu Weichenstellungen distributiver Art (Umschichtung von Haushalts- und Strukturfondsmitteln; Kredite der Europäischen Investitionsbank [EIB]; projektiertes Volumen von $6 \mathrm{Mrd}$ € bis 2020) und "normativer" Art (Proklamation einer „Jugendgarantie“). Auch fördert die EU die Arbeitsmobilität durch Maßnahmen, die eine bessere Information und Unterstützung bei der Arbeitssuche im Ausland und einen leichteren Zugang zur Arbeitslosenunterstützung im Ausland gewährleisten. In jenen Bereichen, in denen die EU zur arbeits- und sozialpolitischen Regulation vertraglich ermächtigt wäre, kam es im Krisenverlauf zu keiner Rechtsetzung. Gleiches gilt auch für Politikfelder, die an die Sozial- und Beschäftigungspolitik angrenzen bzw. diese mittel- und unmittelbar be- 
einflussen, etwa eine gemeinsame Unternehmensbesteuerung. Auch die Einführung einer EU-Finanztransaktionssteuer ist politisch blockiert.

Betrachtet man die Steuerungsbereiche, in denen statt "harter" Regulierung überwiegend mit „weichen“ Mechanismen gearbeitet und soft law hervorgebracht wird, also die Offene Methode der Koordinierung und die EUSozialdialoge, so sind auch hier keine krisenbedingten Veränderungen zu beobachten, die über deren bisherige Steuerungsqualitäten hinausgreifen würden.

So konnte die potenzielle institutionelle Machtquelle des (Multi-)Sektoralen Sozialdialogs (es existieren derzeit 42 sog. Ausschüsse des Sektoralen Sozialdialogs) im Verlauf der Krise keine unmittelbaren Regulierungs- oder Steuerungsfunktionen erfüllen, die zugleich Ausdruck qualitativ intensivierter Arbeitsbeziehungen auf EU-Ebene wären (Rüb/Platzer 2015, S. 201ff.). Immerhin wird dem Sozialen Dialog der Europäischen Gewerkschafts- und Arbeitgeberdachverbände in den makro-ökonomischen Koordinierungsverfahren und im Rahmen des Europäischen Semesters ein größeres Gewicht beigemessen. Damit wird gewerkschaftlichen Forderungen nach einer stärkeren Partizipation (zumindest prozedural) Rechnung getragen. Ansatzweise Gehör findet auch ein vom Deutschen Gewerkschaftsbund (DGB) bereits 2012 entwickeltes und vom Europäischen Gewerkschaftsbund (EGB) übernommenes Konzept eines „Marschallplans für Europa“, das eine Investitionsoffensive im Bereich der europäischen Infrastruktur vorsieht. Auf politscher Ebene sind mit dem von Kommissionspräsident Juncker initiierten „Investitionsfonds“ Weichen in diese Richtung gestellt worden: Durch eine „Anschubfinanzierung“ aus öffentlichen EU-Mitteln in der Größenordnung von rund 20 Mrd. $€$ sollen private Investitionen in mehr als zehnfacher Höhe generiert werden.

Die jüngste sozialpolitische Innovation stellt die im April 2017 proklamierte Europäische Säule sozialer Rechte (ESSR) dar. Sie soll nach Jahren eines überwiegend neoliberalen Krisenmanagements der sozialen Dimension der Europäischen Union zu mehr Sichtbarkeit verhelfen und als Kompass für eine Aufwärtskonvergenz in Richtung besserer Arbeits- und Lebensbedingungen in Europa dienen. Mit der Säule, die sich in erster Linie an den Euro-Raum richtet, werden 20 zentrale Grundsätze und Rechte festgeschrieben, die in drei Kategorien unterteilt sind: Chancengleichheit und Arbeitsmarktzugang, faire Arbeitsbedingungen sowie Sozialschutz und soziale Inklusion.

Überwiegend fasst die Säule den sozialen Rechtsbestand der EU lediglich zusammen. In einigen Punkten geht sie aber auch darüber hinaus. Unter anderem wird ein Recht auf Mindesteinkommensleistungen sowie ein Anspruch auf angemessene Mindestlöhne formuliert. Die Verwirklichung der in der ESSR festgeschriebenen Grundsätze und Rechte soll in der gemeinsamen Verantwortung von Mitgliedstaaten, EU-Institutionen, Sozialpartnern und sonstigen Akteuren liegen. Unter Wahrung der $\mathrm{Zu}$ ständigkeiten der Mitgliedstaaten und der nationalen tarifpolitischen Traditionen sollen auf europäischer Ebene der erforderliche Rahmen abgesteckt und die Richtung zur Umsetzung der Säule vorgegeben werden. Die Haltung der Gewerkschaften zur ESSR spiegelt ein Dilemma: Das teils in den Inhalten, teils in den Umsetzungsverfahren als unzulänglich kritisierte Dokument wird letztlich begrüßt, weil dadurch die soziale Dimension überhaupt wieder auf die politische Agenda gehoben wird. Erstmals wurde die ESSR mithilfe des Social Scoreboards in das Europäische Semester 2017/2018 eingebunden. Eine erste Evaluation von Hacker (2018) kommt zu einem eher ernüchternden Ergebnis: Demnach wird das Potenzial des begleitenden Social Scoreboards zur Kenntlichmachung sozialer Missstände in der EU nur unzureichend genutzt: Zum einen zeigten sich die Mitgliedstaaten reserviert, zum anderen gelinge keine Korrektur der Dominanz budgetärer und wettbewerbsbezogener gegenüber sozialen Zielen.

Insgesamt betrachtet haben das Krisenmanagement und das mittlerweile etablierte System der Economic Governance im Zusammenspiel ordnungs-, integrationsund demokratiepolitischer Faktoren zu einer tendenziellen Schwächung der institutionellen gewerkschaftlichen Machtressourcen im EU-Mehrebenensystem geführt. Ordnungspolitisch dominierten im Krisenverlauf eine neoliberale und austeritätspolitische Wirtschaftsphilosophie. Integrationspolitisch wird die grundlegende Asymmetrie zwischen den „marktschaffenden“ und „marktkorrigierenden “ EU-Politiken fortgeschrieben. Während die inhärente Wettbewerbslogik der neuen Economic Governance, die EU-Mitgliedstaaten in ein Konkurrenzverhältnis zueinander setzt und soziale Konflikte (re-)nationalisiert (Erne 2015, S.353), sind (bislang) auf europäischer Ebene allenfalls bescheidene politische und gesetzgeberische Innovationen hervorgebracht worden, die als „sozialund arbeitspolitischer Flankenschutz" die transnationale gewerkschaftliche Partizipation und Interessenartikulation institutionell stärken. Auch demokratiepolitisch kommt es zu keiner Stärkung supranationaler Entscheidungsverfahren mit einem entsprechend gewichtigeren Europäischen Parlament (und einer potenziell gestärkten Rolle der EGV), da das neue wirtschafts- und währungspolitische Instrumentarium vor allem auf zwischenstaatlichen Verträgen und mithin auf nationalen und intergouvernementalen Steuerungslogiken beruht.

3.2.3 Transnationale organisatorische Machtressourcen Betrachtet man anhand ausgewählter Organisationsdaten den Entwicklungsstand der europäischen Gewerkschaftsföderationen zu Beginn und am Ende des Krisenjahrzehnts, so ergibt sich das in Übersicht 3 dargestellte Bild.

Die personelle Ausstattung der Sekretariate ist bei allen Verbänden vergleichsweise konstant geblieben. Mit Ausnahme des Dachverbandes EGB ist bei den meisten EGV auch die repräsentierte Gesamtmitgliedschaft relativ 
ÜBERSICHT 3

\section{Strukturdaten der Europäischen Gewerkschaftsverbände, 2008 und 2018}

Angaben in absoluten Zahlen und in Millionen

\begin{tabular}{|c|c|c|c|c|c|c|c|c|}
\hline \multirow[t]{2}{*}{$\begin{array}{l}\text { Europäischer } \\
\text { Gewerkschaftsverband }\end{array}$} & \multicolumn{2}{|c|}{$\begin{array}{l}\text { Repräsentierte Gesamt- } \\
\text { mitgliedschaft in Millionen }\end{array}$} & \multicolumn{2}{|c|}{ Mitgliedsverbände } & \multicolumn{2}{|l|}{ Länder } & \multicolumn{2}{|c|}{$\begin{array}{l}\text { Personal } \\
\text { am Sitz des Sekretariats }\end{array}$} \\
\hline & 2008 & 2018 & 2008 & 2018 & 2008 & 2018 & 2008 & 2018 \\
\hline EGB & 60 & 45 & 86 & 89 & 39 & 39 & $46^{A}$ & 61 \\
\hline EGÖD & 8 & 8 & 209 & 265 & 36 & 49 & 16 & 19 \\
\hline UNI-Europa & 7 & 7 & 320 & 272 & 50 & 50 & k. A. & 23 \\
\hline EGBW & 5,5 & 11 & 112 & 132 & 30 & 51 & 10 & 12 \\
\hline industriAll Europe ${ }^{B}$ & & 7 & & 192 & & 37 & & 24 \\
\hline EMB & 5,4 & & 69 & & 33 & & 20 & \\
\hline EMCEF & 2,5 & & 128 & & 35 & & 8 & \\
\hline EGV:TBL & 1 & & 70 & & 40 & & 4 & \\
\hline EFFAT & 2,6 & 2,6 & 120 & 120 & 35 & 35 & 11 & 11 \\
\hline ETF & 2,5 & 5 & 223 & 230 & 40 & 42 & 14 & 16 \\
\hline EFBH & 2,3 & 2 & 68 & 76 & 27 & 34 & 8 & 9 \\
\hline EuroCOP & 0,530 & 0,530 & 34 & 35 & 26 & 27 & $1-2$ & 2 \\
\hline EAEA & 0,300 & k. A. & 135 & k. A. & 27 & k. A. & k. A. & k. A. \\
\hline EJF & 0,280 & 0,320 & 53 & 70 & 34 & 44 & 4 & 6 \\
\hline
\end{tabular}

A Zahl für 2006

B industriAll Europe entstand 2012 als Zusammenschluss von EMB, EMCEF und EGV:TBL.

$E G B=$ Europäischer Gewerkschaftsbund, EGÖD $=$ Europäischer Gewerkschaftsverband für den Öffentlichen Dienst, UNI-Europa $=$ Union Network International - Europa, EGBW $=$ Europäisches Gewerkschaftskomittee für Bildung und Wissenschaft, industriAll = European Trade Union, EMB = Europäischer Metallgewerkschaftsbund, EMCEF $=$ Europäische Föderation der Bergbau-,

Chemie- und Energiegewerkschaften, EGV:TBL = Europäischer Gewerkschaftsverband Textil, Bekleidung und Leder, EFFAT = Europäische Föderation der Gewerkschaften für den Lebensmittel-,

Landwirtschafts- und Tourismussektor, ETF = Europäische Transportarbeiter-Föderation, EFBH = Europäische Föderation der Bau- und Holzarbeiter, EurocOP $=$ Europäische Föderation der Polizei,

$\mathrm{EAEA}=$ Europäische Allianz für Kunst und Unterhaltung, EFJ = Europäische Journalisten-Föderation

Quelle: Zusammenstellung der Autoren auf Grundlage von Angaben der Organisationen

stabil geblieben. Bei zwei Verbänden, dem EGBW und der ETF, hat sie sich verdoppelt. Bei der Mehrzahl der EGV ist die Anzahl der nationalen Mitgliedsverbände und der repräsentierten Länder (leicht) angestiegen. Alles in allem signalisieren diese Zahlen zunächst eine gewisse Kontinuität und relative Stabilität der transnationalen gewerkschaftlichen Organisationsbedingungen. Freilich gilt es zu bedenken, dass mit dem Zuwachs meist kleiner Verbände aus weiteren Ländern (meist der europäischen Peripherie) die mitgliedschaftliche Heterogenität der EGV zunimmt und die ohnehin schon schwierige Aufgabe der Interessenaggregation und der Koordinierung der Aktivitäten der Mitgliedsbünde noch anspruchsvoller wird. Schwierigkeiten ergeben sich vor allem mit Blick auf die unterschiedlichen gewerkschaftlichen Belange aus EU- und Nicht-EU-Ländern, aber auch mit Blick auf ein Europa der „unterschiedlichen Geschwindigkeiten“, weil sich beispielsweise tarif-und sozialpolitische Fragen für Gewerkschaften innerhalb der Euro-Zone anders stellen als für Gewerkschaften außerhalb.

3.2.4 Transnationale kommunikative Machtressourcen Die ökonomische und fiskalische „Philosophie“ und die Regelwerke der neuen European Economic Governance zeigen, dass es den Gewerkschaften trotz nationaler Bei- spiele eines vergleichsweise erfolgreichen „Krisenkorporatismus" im Verlauf der Krise insgesamt nicht gelungen ist, ihre wirtschaftspolitischen Vorstellungen und Lösungskonzepte im EU-Mehrebenensystem durchzusetzen. Insbesondere in der „Hochphase der Krise“ war ein kohärentes transnationales Gewerkschaftshandeln erschwert, da die politische Mobilisierung einen Gegnerbezug voraussetzt und dieser in Anbetracht unterschiedlicher Krisendeutungen und Konflikteinordnungen nicht einfach herzustellen war. „Während beispielsweise für griechische und spanische Gewerkschaftsmitglieder neben der eigenen Regierung die EU und die Troika sowie möglicherweise noch die bundesdeutsche Kanzlerin klar ausmachbare Gegner darstellen, an die sie ihre Forderungen richten können, stellt sich dies für bundesdeutsche Gewerkschafter nicht so eindeutig dar. Von der Troika geht keine direkte Bedrohung aus, und das vorgebliche Bemühen der deutschen Europapolitik, die Belastungen für deutsche Steuerzahler so gering wie möglich zu halten, findet durchaus Zustimmung" (Dribbusch 2014, S. 342).

Neben diesen politisch-kommunikativen Barrieren bestand innerhalb des europäischen Gewerkschaftslagers auch keine ausreichend homogene wirtschaftliche Interessenbasis, um die gewerkschaftliche Marktmacht transnational aggregiert in Anschlag zu bringen. Unter diesen 
Vorzeichen lag auch eine europaweite nachhaltige Streikbewegung nicht im Rahmen des Möglichen. Realisiert werden konnten einige zwischenstaatlich abgestimmte gewerkschaftliche Solidaritätsaktionen sowie Massendemonstrationen und Protestaktionen am Sitz der EU-Organe in Brüssel.

In den Jahren nach 2012 und mit Antritt der Juncker-Kommission gelang es den Gewerkschaften, ihre kommunikativen Machtressourcen auf europäischer Ebene etwas stärker zur Geltung zu bringen. Das heißt, die Gewerkschaften konnten, stärker als zu Beginn der Krise, mit programmatisch präzisierten Vorstellungen und transnational abgestimmten politischen Forderungen ${ }^{2}$ auf die politische Agenda einwirken und wenigstens in einzelnen Bereichen Erfolge verbuchen. ${ }^{3}$

Diese graduellen Errungenschaften sind freilich nur in Teilen auf den Einsatz gewerkschaftlicher Machtquellen zurückzuführen. Sie hängen wesentlich auch damit zusammen, dass die 2015 ins Amt gekommene Juncker-Kommission erkannt hatte, dass ohne neue Akzente im Bereich eines „sozialen Europa“ eine fortschreitende Delegitimierung des Integrationsprojekts drohte. Auch wenn das von Juncker proklamierte ehrgeizige Ziel, die EU in den $\mathrm{Zu}$ stand eines „sozialen AAA-Ratings“ zu befördern, in weiter Ferne liegt, haben diese politischen Akzentverschiebungen ein Diskussionsklima geschaffen, in dem gewerkschaftliche Ideen etwas besser gedeihen konnten.

\section{Befunde und Ausblicke}

Wo steht die europäische Gewerkschaftsbewegung eine Dekade nach Ausbruch der Krise und wie haben sich in Folge der Krise die gewerkschaftlichen Machtressourcen im EU-Mehrebenensystem und die Funktionsprofile der EGV verändert?

Angesichts der Tatsache, dass es sich um die tiefste globale Krise seit der Weltwirtschaftskrise der 1920er Jahre gehandelt hat und zugleich als „Euro-Krise“ um die tiefste Krise der EU seit deren Bestehen, wären zwei Extremszenarien denkbar gewesen, die jedoch nicht eingetroffen sind: Weder ist es zu einer Entwicklung gekommen, in der die transnationale Zusammenarbeit infolge nationaler Souveränitätsreflexe und zentrifugaler Tendenzen vollständig blockiert gewesen wäre und die europäischen Gewerkschaftsorganisationen erodiert wären. Noch ist eine Entwicklung eingetreten, in der die gemeinsamen Erfahrungen einer neuen Qualität wechselseitiger Verwundbarkeit und eines damit verbundenen transnationalen Solidaritätsbedarfs zu einer neuen Stufe der gewerkschaftlichen Europäisierung geführt hätten.

Gegenüber diesen (denkbaren) Extremszenarien eines weitreichenden Rückbaus oder substanziellen Ausbaus der transnationalen Gewerkschaftspolitik ist es in der Organisation und Politik der Europäischen Gewerkschaftsverbände über das Krisenjahrzehnt hinweg alles in allem nur zu graduellen Veränderungen gekommen, sodass sich ihr heutiger Entwicklungsstand im Vergleich zur „Vorkrisenzeit" um einen Status quo ante herum bewegt.

Diesen generellen Befund gilt es allerdings entlang der Zeitachse und nach gewerkschaftlichen Handlungsfeldern zu differenzieren. Beispielsweise waren die EGV in der „Hochphase“ der Euro-Krise nicht mehr in der Lage, die zuvor erreichten transnationalen Koordinierungsleistungen im zentralen Handlungsfeld der Tarifpolitik aufrechtzuerhalten. Die Einhaltung der von den meisten EGV verabschiedeten Lohnkoordinierungsregel, wonach Lohnsteigerungen mindestens die Inflation und den Anstieg der Produktivität abdecken sollten, war für viele Gewerkschaften schon vor der Krise ein schwieriges Unterfangen. Unter den Bedingungen des krisenbedingten ökonomischen Regimes der Austerität und der internen Abwertung in den Krisenländern wurde dies noch schwieriger und teilweise unmöglich. Es bleibt abzuwarten, ob es den EGV in diesem Bereich gelingt, ihr zwischenzeitlich zurückgeworfenes Funktionsprofil wieder in Richtung einer effizienten Koordinierungsplattform zu treiben. In anderen Handlungsfeldern war die Politik der EGV durch die Fortschreibung von Verbandsroutinen gekennzeichnet. Dies gilt etwa für die Beratung und Begleitung von Europäischen Betriebsräten, für die Akquise von EU-Mitteln zur Förderung von arbeitspolitischen Qualifizierungsmaßnahmen oder für die Interessenvermittlung in den Ausschüssen des Sektoralen Sozialdialogs.

Im gegenwärtigen Europa der sich mehrfach überlagernden Krisen (instabile Finanzmärkte, soziale Problemlagen, Migration, Brexit, wachsender Rechtspopulismus, Bedrohung der Demokratie in einzelnen EU-Mitgliedstaaten) steht die europäische Gewerkschaftsbewegung vor gewaltigen Herausforderungen. Auf der europäischen Ebene der EGV gilt es, schwierige programmatische und strategische Richtungsentscheidungen $\mathrm{zu}$ treffen. Vor dem Hintergrund veränderter, teils geschwächter Machtressourcen und einer tendenziell gewachsenen Heterogenität nationaler Gewerkschaftsinteressen gestaltet sich dieser transnationale Willensbildungsprozess gegenwärtig aus mehreren Gründen weit schwieriger als in vorangegangenen Entwicklungsphasen der europäischen Integra-

2 Hierzu zählen bspw. der 2013 vorgelegte Investitionsplan des EGB oder die von industriAll und UNI Europa 2014 entwickelten europäischen Initiativen zur Stärkung von Wachstum und hochwertigen Arbeitsplätzen.

3 Dazu zählen etwa die Ansätze einer nachfragorientierten EU-Investitionspolitik, die stärkere Normierung sozial- und arbeitspolitscher Belange durch die ESSR oder die intensivere gewerkschaftliche Mitbeteiligung in den Beratungsund Steuerungsprozessen des Europäischen Semesters. 
tion: Zum einen sind konsensfähige gewerkschaftliche Kernanliegen, wie die Korrektur der sozialen Fehlentwicklungen einer wirtschaftsliberalen Europäisierung durch eine wirtschafts- und gesellschaftspolitische Neuausrichtung der EU-Politik, nicht zu trennen von gemeinsamen Reformvorstellungen zur institutionellen Ordnung und Kompetenzausstattung der EU, also von Fragen, die zwischen den nationalen Gewerkschaften traditionell eher strittig sind. Zum anderen ist eine europäische Interessenbündelung oder gar eine prointegrative Mobilisierung für die Gewerkschaften gegenwärtig ein besonders schwieriges Geschäft, weil das Thema
Europa an der Mitgliederbasis eine eher nachrangige Bedeutung hat und zugleich Renationalisierungsbotschaften der „Internationale der Nationalisten“ und der europaweit stärker werdenden populistischen Kräfte auch unter Gewerkschaftsmitgliedern Resonanz finden.

Unter diesen Vorzeichen ein Funktionsprofil der Europäischen Gewerkschaftsverbände (wieder-)zuerlangen bzw. aufrechtzuerhalten, das (zumindest) die Qualität einer effektiven Koordinierungsplattform besitzt, ist ein ebenso schwieriges wie unverzichtbares Unterfangen.

\section{LITERATUR}

Dribbusch, H. (2014): Voraussetzungen internationaler Solidarität: zur Dis kussion um einen europäischen Generalstreik, in: WSI-Mitteilungen 67 (5) S. 337-344, https://www.boeckler.de/wsimit_2014_05_dribbusch.pdf

Erne, R. (2015): A supranational regime that nationalizes social conflict: explaining European trade unions' difficulties in politicizing European economic governance, in: Labor History 56 (3), S. 345-368

Hacker, B. (2018): Soziales Europäisches Semester? Die Europäische Säule sozialer Rechte im Praxistest: Institut für Europäische Politik, IEP-Research Paper No. $02 / 18$

Haipeter T./ Dörre K. (Hrsg.) (2011): Gewerkschaftliche Modernisierung, Wiesbaden

Huke N. / Tietje O. (2015): Zwischen Kooperation und Konfrontation. Machtressourcen und Strategien der spanischen Gewerkschaften CCOO und UGT in der Eurokrise, in: Industrielle Beziehungen 21 (4), S. 371-389

Müller, T./ Platzer, H.-W. (2016): Gewerkschaften und Arbeitsbeziehungen im Europa der Krise: Strukturdaten und Entwicklungstrends im Vergleich, in: Bsirske, F. / Busch, K. / Höbel, O. / Knerler, R. / Scholz, D. (Hrsg.): Gewerkschaften in der Eurokrise: Nationaler Anpassungsdruck und europäische Strategien, Hamburg,

S. $10-44$

Müller, T. / Platzer, H.-W. (2018): The European trade union federations: profiles and power resources - changes and challenges in times of crisis, in: Lehndorff, S. / Dribbusch, H. / Schulten T. (eds.): Rough waters - European trade unions in a time of crisis, Brussels

Platzer, H.-W. / Müller, T. (2009): Die globalen und europäischen Gewerkschaftsverbände. Handbuch und Analysen zur transnationalen Gewerkschaftspolitik (unter Mitarbeit von Rüb, S. / Helmer, M. / Oettgen, T. R.), Berlin

Rüb, S./ Platzer H.-W. (2015): Europäisierung der Arbeitsbeziehungen im Dienstleistungssektor. Empirische Befunde, Probleme und Perspektiven eines heterogenen Feldes, Berlin

Schmalz S./ Dörre K. (Hrsg.) (2013): Comeback der Gewerkschaften? Machtressourcen, innovative Praktiken, internationale Perspektiven, Frankfurt a. M.

Schulten T./ Müller T. (2015): European economic governance and its intervention in national wage development and collective bargaining, in: Lehndorff, S. (Hrsg.): Divisive integration: the triumph of failed ideas in Europe - revisited, Brussels, S. 331- 363

\section{AUTOREN}

HANS-WOLFGANG PLATZER, Dr., ist Professor (i. R.) an der Hochschule Fulda und Inhaber eines Jean Monnet Chair of European Integration. Forschungsschwerpunkte: Europäische Integration/Europäische Wirtschafts- und Sozialpolitik.

hans.w.platzer@sk.hs-fulda.de

TORSTEN MÜLLER, Dr., ist Senior Researcher beim Europäischen Gewerkschaftsinstitut in Brüssel. Forschungsschwerpunkte: Tarifpolitik und Arbeitsbeziehungen in Europa.

tmueller@etui.org 